\title{
Using Complex-Valued Levenberg-Marquardt Algorithm for Learning and Recognizing Various Hand Gestures
}

\author{
Abdul Rahman Hafiz*, Md Faijul Amin*and Kazuyuki Murase* \\ *Department of Human and Artificial Intelligence System, \\ Graduate School of Engineering, \\ University of Fukui, JAPAN \\ Email: murase@synapse.his.u-fukui.ac.jp
}

\begin{abstract}
With the advancement in technology, we see that complex-valued data arise in many practical applications, specially in signal and image processing. In this paper, we introduce a new application by generating complex-valued dataset that represents various hand gestures in complex domain. The system consists of three components: real time hand tracking, handskeleton construction, and hand gesture recognition. A complexvalued neural network (CVNN) having one hidden layer and trained with Complex Levenberg-Marquardt (CLM) algorithm has been used to recognize 26 different gestures that represents English Alphabet. The result shows that the CLM provides reasonable recognition performance. In addition to that, a comparison among different activation functions have been presented.
\end{abstract}

\section{INTRODUCTION}

Recent technological advancements in the human-computer interaction field have shown that conventional tools, such as keyboard, mouse, and light pen, do not provide a natural form of interaction. Even though those tools were the standard forms of input for many decades, the ubiquity of digital systems revealed the urgent requirement for a more reachable interaction method that can be used by anyone regardless of his educational background. Since the hand has always been the natural interaction methods among humans, a recent resurgence in developing new hand modeling techniques has been observed. Regardless of the technique used, the main goals have always been: using descriptive gestures while keeping the computer processing and modeling as simple as possible.

The system can be applied to various background, changeable lighting of the environment and different kinds of human colors. To achieve that, we construct simple representation of human hand (hand-skeleton) after applying edge detection with thinning algorithm (Fig.1) to the input image, we then define gestures for each English characters [1].

Recently, complex-valued data are used in many applications, such as array signal processing [2], radar and magnetic resonance data processing [3], [4], communication systems [5], signal representation in complex baseband [6], and processing data in the frequency domain [3].

In our approach, complex-valued data that represent hand gesture can be obtained after applying sequence of filters to
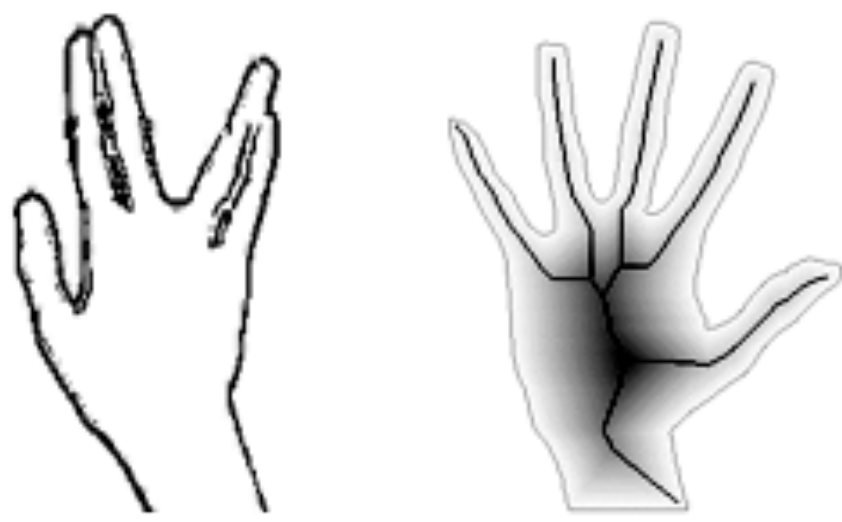

Fig. 1. The left image is a human hand after applying edge detection algorithm, while the image on the right is showing the branches that were produced after applying thinning algorithm to the image

the image captured using Kinect camera [7]. We used three layer complex-valued neural network (CVNN), and Complex Levenberg-Marquardt (CLM) algorithm [8] for the training, due to the nature of the data that we can collect from the generated hand-skeleton representation. We investigate the recognition performance with respect to various activation functions in the hidden layer. The output layer, however, uses a recently proposed activation function [11], that helps an output neuron behaving like a discriminative function.

The remainder of the paper is organized as follows. Section II, discusses the procedures to generate the hand-skeleton structure. Section III introduces CLM algorithm and various activation functions. Computer simulation results are discussed in Section IV. Finally, concluding remarks are given in Section V.

\section{Procedures}

In this research, we utilized the camera of a Microsoft Kinect motion-sensing input device [7], accompanied by OpenCV platform which have the computational capabilities required for real-time image acquisition and handling. Fig. 
2 represents a modular view of the final system. The image acquired by the Video Input Module is passed to both the Hand Location Module and the Image Processing Module. While the Hand Location Module is responsible for detecting the location of the hand within the image, the Image Processing Module processes the area that has been previously detected by the Hand Location Module. The output of the Image Processing Module is then passed to the Hand-Skeleton Construction Module which is responsible for creating a skeleton module from the image of the hand. This module has two outputs: skeleton model of the hand, and supplementary data passed to the Hand Location Module to increase the location detection accuracy. The skeletal model is then passed to the CLM where the actual recognition takes place. The following subsections describe in detail each stage of the system:

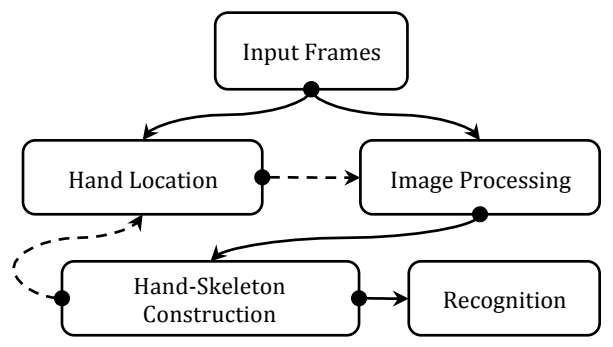

Fig. 2. Human hand gesture recognition system, showing its module and the connections between them

\section{A. Tracking and Detection}

The first step involves separating the image of the hand from the rest of the image. To do that, we used Kinect's depth map to wipe the background of the image. As we can see in Fig. 3. , only the silhouette of the human body was extracted from the image, disposing any other unneeded objects.

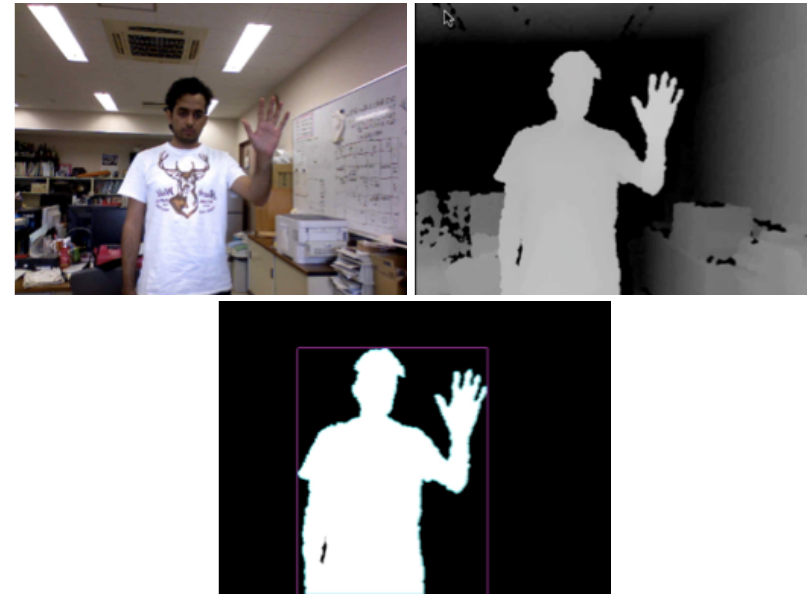

Fig. 3. Simple illustration of background deletion, the image in the left is the source image, the image in the middle is the depth map that Kinect's camera provides, and the image in the right is the result after deleting the background

Next, we removed the regions not having the color of human skin. The resulting image contains the location of the human hand and face. Then we used HSL representation of color to identify the color of human skin. It is known that HSL representation identifies the color of human skin more accurately than RGB representation[12]. The last step involved separating the image of the hand from image of the face (Fig. 4).
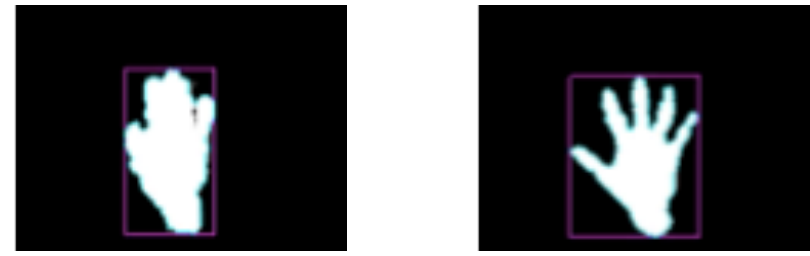

Fig. 4. The result after skin detection, showing human hand with two states. In the left, the human hand with the fingers are together, and in the right, the fingers are apart

Although the hues of the hand's color and the face's color are different, the difference is too small to be considered reliable by itself. Accordingly, we had to support that by another source of information. For instance, when the system is initialized, it depends on the motion of the hand to distinguish it from the face, and then the system would realize the location of the hand using the feed-back of the hand-skeleton construction part of the system.

From Fig. 4, we can notice that when the fingers are close to each other, we might lose some information about each finger state. To compensate for that problem, we used a sequence of image processing algorithms to aid the correct recognition of the finger's state, as described in the next section.

\section{B. Image Processing}

After locating the human hand in the image, the system filters the region where the hand is located as shown in Fig. 5.

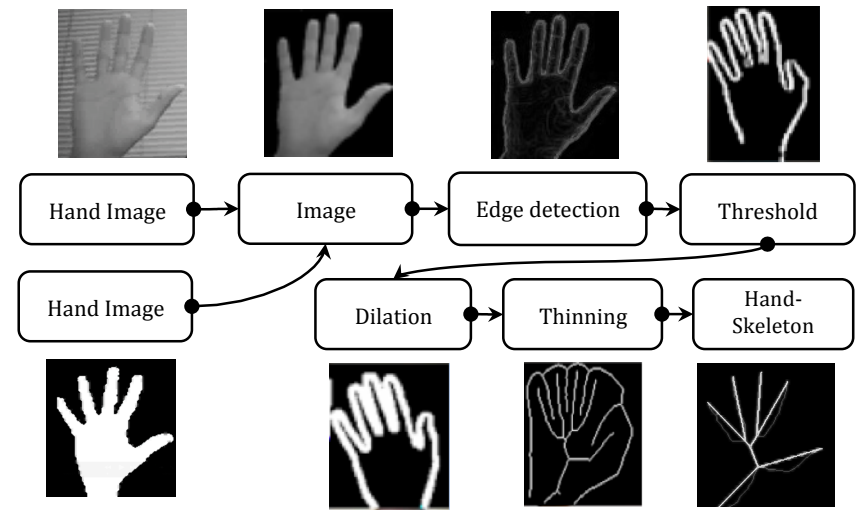

Fig. 5. The image filters that applied in real-time for the hand location, that produce connected branches of line which represent the fingers of human hand

First, the system applies Sobel Edge Detection Algorithm [10] to get a contour of the hand. This filter scans the image for sharp contrast differences, and assigns a white color shade equivalent to the contrast in that region. 
Next, by restricting the whiteness to a specific threshold, the system deletes any noisy edges effectively creating a sharper edge representation. However, that step would produce disconnected regions in the edges of the hand, affecting the outcome of thinning algorithm. To avoid that drawback, we used a dilation algorithm resulting in a fully connection figure.

The dilated image is then passed to a thinning algorithm [8]. This algorithm generates one line of pixels representing the branching of the structure. The outcome of that step is: a representing the hand as interconnected lines meeting at multiple nodes.

The final step involves reading that representation. The system creates pairs of data for each branching by traces the line that connects the nodes, calculating the length and the angle of these lines. The calculation method for the length of the line which connects two nodes and its relative angle is shown in Fig. 6.

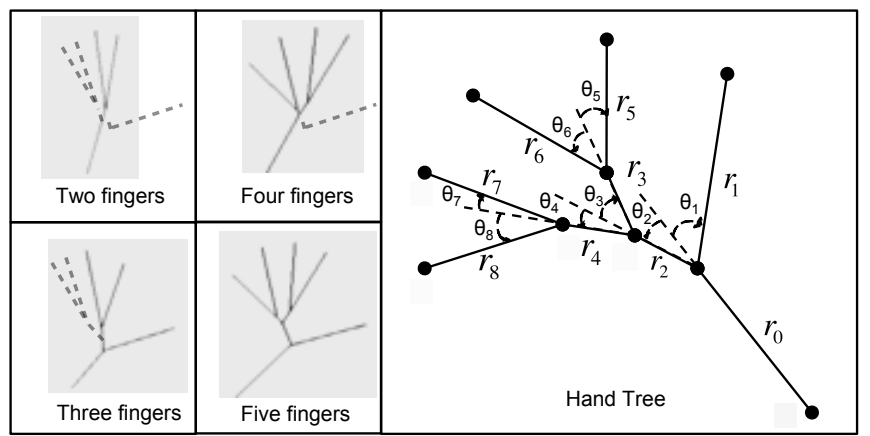

Fig. 6. The left side of the figure is the hand-skeleton branches and nodes that have been generated from the result of the thinning algorithm. The right side is the pairs of data that can be abstracted from these branches

Fig. 6 shows the method used for converting a hand-skeleton model to a set of amplitude-phase pairs that can be processed by the CVNN. The conversion process involves the recursive measurement of the branches relative to the root branches. The complete conversion process is made of the following steps:

1) Locate the lower-most root branch.

2) Measure the angle between branches from the node at the end of the root branch and the extension of the root branch $\left(\theta_{1}\right)$ and the length of that branch $\left(r_{1}\right)$.

3) Repeat step 2 until you reach branches that don't branch at their terminal nodes.

4) Input the parameters of the non-branching branches (terminal branches) to the CVNN

5) In the case when the number of terminal branches is not equal to 5 (that is, less than the number of human fingers), other terminal branches are assigned with zeroes for both phase and amplitude. For simplicity, the assumed pictures will follow a predefined pattern, as can be seen in Fig. 6 .

\section{Complex-VAlued LeVenberg-Marquardt} (CLM) Algorithm FOR Hand Gesture ReCOGNition

The image processing steps discussed above produces a complex vector $\mathbf{x}=\left[x_{1}, x_{2}, \ldots, x_{8}\right]^{T}$ consisting of eight elements. Each element $x_{i}, 1 \leq i \leq 8$, is a Cartesian representation of each segment of the hand skeleton, i.e.e., $x_{i}=r_{i} e^{j \theta_{i}}=r_{i} \cos \left(\theta_{i}\right)+j r_{i} \sin \left(\theta_{i}\right)$, where $j=\sqrt{-1}$. To classify the patterns represented by complex-valued feature vectors, we apply a feedforward complex-valued neural network (CVNN) with one hidden layer. The output layer uses an activation function proposed in [11] which can act as a discriminating function giving a discriminating score. We call the function here as discrim. The function has the following form:

$$
f_{\mathbb{C} \rightarrow \mathbb{R}}(z)=\left(f_{R}(u)-f_{R}(v)\right)^{2}
$$

where $z=u+j v$ denotes the weighted sum of input signals along with the bias and called net-input and $f_{R}()$ is a realvalued log-sigmoid function. The hidden layer, however, may take any activation function found in the literature of the CVNNs. Recently CLM has been proposed by [8] as a fast learning algorithm for the feedforward complex-valued neural networks (CVNN). The CVNN in this study is trained with the CLM algorithm because of its faster convergence. Since we have a total of 26 different gestures, the output layer has 26 neurons, each representing one gesture.

In order to see the effect of hidden layer activation functions in the hand gesture recognition problem, we investigate a number of complex activation functions listed below.

splitTanh [14]: $\quad f(z)=\tanh u+j \tanh v$

splitSigm [14] : $\quad f(z)=\frac{1}{1+e^{-u}}+j \frac{1}{1+e^{-v}}$

linear: $f(z)=z$

George [11]: $\quad f(z)=z /(c+|z| / r), c, r$, constants

$\tan [15]: \quad f(z)=\frac{e^{i z}-e^{-i z}}{i\left(e^{i} z+e^{-i z}\right)}$

$\sin [15]: \quad f(z)=\frac{e^{i z}-e^{-i z}}{2 i}$

$\operatorname{atan}[15]: \quad f(z)=\arctan z=\int_{0}^{z} \frac{d t}{1+t^{2}}$

$\operatorname{asin}[15]: \quad f(z)=\arcsin z=\int_{0}^{z} \frac{d t}{(1-t)^{1 / 2}}$

$\operatorname{acos}[15]: \quad f(z)=\arccos z=\int_{0}^{z} \frac{d t}{\left(1-t^{2}\right)^{1 / 2}}$

$\sinh [15]: \quad f(z)=\frac{e^{z}-e^{-z}}{2}$

$\tanh [15]: \quad f(z)=\frac{e^{z}-e^{-z}}{e^{z}+e^{-z}}$

$\operatorname{atanh}[15]: \quad f(z)=\operatorname{arctanh} z=\int_{0}^{z} \frac{\mathrm{d} t}{\left(1+t^{2}\right)^{1 / 2}}$

asinh [15] : $\quad f(z)=\operatorname{arcsinh} z=\int_{0}^{z} \frac{\mathrm{d} t}{(1-t)^{1 / 2}}$

\section{RESUlTS}

A pattern set with 26 hand gestures were collected, The data set comprises 520 patterns and was divided into a training set $(50 \%)$, a validation $(25 \%)$, and a testing set $(25 \%)$. 
We test the system robustness in simple data collection and noise deletion tasks, the system could work in 10 frames per second (fps) and could read the hand state for $90 \%$ of the time [1].

For recognizing the English character, we defined distinguishable gestures of the hand to represent each character. These gestures have been chosen so that it will be easier for the system to recognize them. Consideration was also taken on human's natural skills to move from one gesture to other. Our algorithm detects the edges between the fingers even though the fingers are stuck together. This allowed us to design a simple representation for each character as shown in Fig. 7.

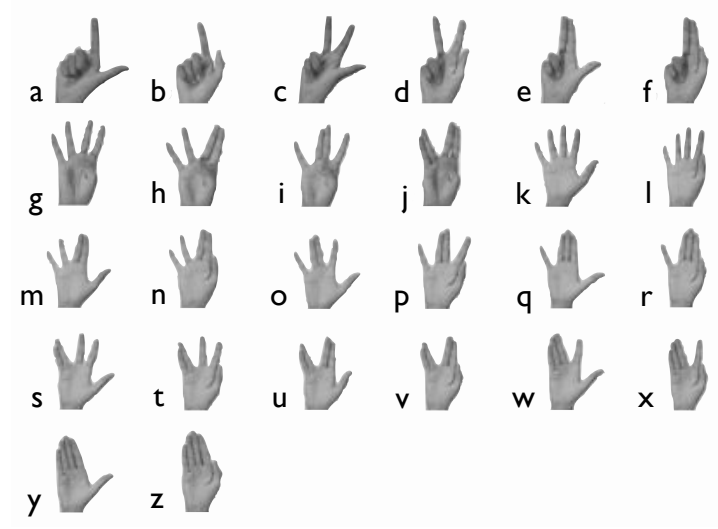

Fig. 7. Hand gesture for each English character, we can notice that the gestures are differing in the number of fingers and the angles they make with each other

We presented all the input data to the CLM and computed the outputs and the validation error. From Fig. 8 we can notice that the optimal number for the neurons in the hidden layer was 4 ,

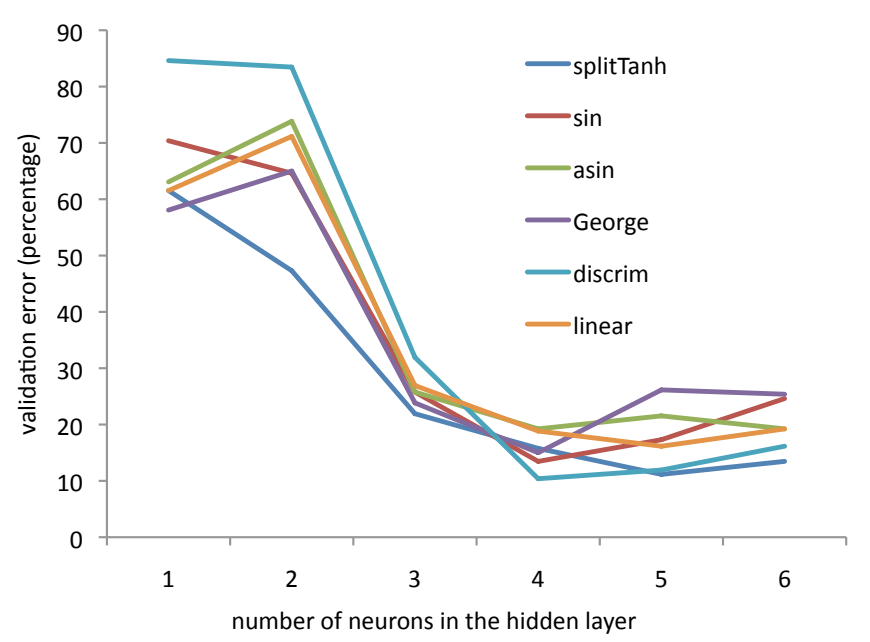

Fig. 8. Validation error for different activations function and number of neurons in the hidden layer

The learning process was terminated if some stopping criteria were met, such as, validation error increases rather than a decrease.

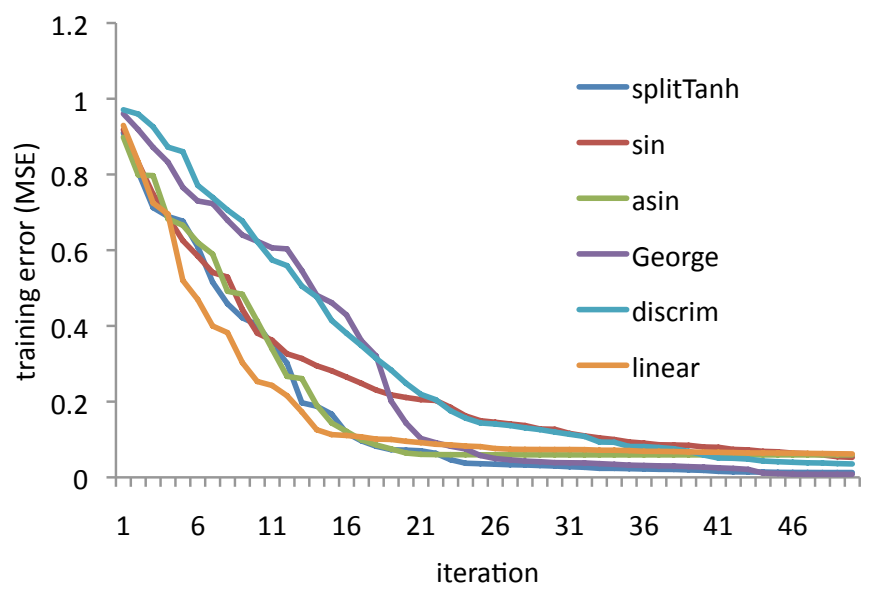

Fig. 9. Mean squared error with different activation functions

Table 1, shows the classification error for different activation functions sorted by the smallest value. From the table we can notice that the split type activation functions performed better for this problem.

TABLE I

CLASSIFICATION ERROR FOR DIFFERENT ACTIVATION FUNCTIONS

\begin{tabular}{|c|c|}
\hline Activation Functions & Classification Error(\%) \\
\hline splitTanh[14] & 08.46 \\
\hline splitSigm[14] & 11.29 \\
\hline linear[15] & 13.59 \\
\hline asin[15] & 15.65 \\
\hline $\tan [15]$ & 16.41 \\
\hline discrim [11] & 17.18 \\
\hline acos[15] & 17.69 \\
\hline $\tanh [15]$ & 17.95 \\
\hline George[13] & 19.49 \\
\hline $\sin [15]$ & 20.00 \\
\hline $\operatorname{asinh}[15]$ & 20.26 \\
\hline $\sinh [15]$ & 21.54 \\
\hline $\operatorname{atan}[15]$ & 23.85 \\
\hline $\operatorname{atanh}[15]$ & 28.97 \\
\hline
\end{tabular}

\section{CONClusion}

In this paper, the CLM algorithm has been used in a hand gesture recognition system to distinguish 26 differed gestures (English Alphabet). By using Kinect depth map and the human skin color we could isolate human hand from the rest of the image, then we used a sequence of image filters to generate a descriptive representation of human hand. We call it "HandSkeleton". This representation allows us to use the CLM algorithm for learning and recognition stage. The results shows that the CLM algorithm with the split type activation functions achieves the highest recognition performance.

\section{ACKNOWLEDGMENT}

This study was supported by grants to K.M. from Japanese Society for promotion of Sciences and Technology, and the University of Fukui. 


\section{REFERENCES}

[1] A. Hafiz, Md.F. Amin and K. Murase, Real-Time Hand Gesture Recognition Using Complex-Valued Neural Network (CVNN), 2011 International Conference on Neural Information Processing (ICONIP 2011), Shanghai, China, Nov. 2011.

[2] H.L. van Trees, Optimum Array Processing, Wiley Interscience, New York, 2002

[3] A. Hirose, An improved parallel thinning algorithm, Springer, Heidelberg, 2006.

[4] V.D. Calhoun, T. Adali, G.D. Pearlson, P.C.M. van Zijl, and J.J. Pekar, Independent component analysis of fMRI data in the complex domain, Magnetic Resonance in Medicine, v.48(1), 180-192, 2002.

[5] G.L. Stuber, Principle of Mobile Communication, Kluwer, Boston, 2001

[6] C.W. Helstom, Elements of Signal Detection and Estimation, Prentice Hall, New Jersey, 1995.

[7] J. Shotton and T. Sharp, Real-Time Human Pose Recognition in Parts from Single Depth Images, published at Microsoft Research in Cambridge, statosuedu, 2, 2011.

[8] Md.F. Amin, I.A. Muhammad ,Y.A.N. Ahmed and K. Murase, Wirtinger Calculus Based Gradient Descent and Levenberg-Marquardt Learning Algorithms in Complex-Valued Neural Networks, 2011 International Conference on Neural Information Processing (ICONIP 2011), Shanghai, China, Nov. 2011.

[9] Intel Corporation, Open Source Computer Vision Library, Reference Manual, Copyright 1999-2001, Available: www.developer.intel.com

[10] I. Sobel and G. Feldman, A $3 \times 3$ Isotropic Gradient Operator for Image Processing, presented at a talk at the Stanford Artificial Project, unpublished but often cited 1968 .

[11] Md.F. Amin, K. Murase, A single-Layered Complex-Valued Neural Network for Real-Valued Classification Problems, Neurocomputing, Vol 72, pp.945-955. 2009.

[12] J. B. Martinkauppi, M. N. Soriano, and M. H. Laaksonen, Behavior of skin color under varying illumination seen by different cameras at different color spaces, in Proc. SPIE, Machine Vision Applications in Industrial Inspection IX, San Jose, CA, Jan. 2001, pp. 102-113.

[13] M.G. Geotge and C. Koutsougeras, Complex domain backpropagation, IEEE Transactions on Circuits and Systems II, 39(5):330-334, 1992.

[14] A. Hirose, Complex-Valued Neural Networks, Springer, 1-160. 2006.

[15] T. Kim and T. Adal, Approximation by fully complex multilayer perceptrons, Neural Computation, vol. 15, no. 7, 1641 pages, 2003. 